\title{
Instrumentation effects on $\mathrm{U}$ and $\mathrm{Pu} \mathrm{CBNM}$ standards spectra quality measured on a 500 $\mathrm{mm}^{3} \mathrm{CdZnTe}$ and a $2 \times 2$ inch $\mathrm{LaBr}_{3}$ detectors
}

\author{
I. Meleshenkovskii, A. Borella, K. Van der Meer, M. Bruggeman, N. Pauly, P.E. Labeau, P. \\ Schillebeeckx
}

\begin{abstract}
Nowadays, there is interest in developing gammaray measuring devices based on the room temperature operated medium resolution detectors such as semiconductor detectors of the CdZnTe type and scintillators of the $\mathrm{LaBr}_{3}$ type. This is true also for safeguards applications and the International Atomic Energy Agency (IAEA) has launched a project devoted to the assessment of medium resolution gamma-ray spectroscopy for the verification of the isotopic composition of $U$ and $P u$ bearing samples. This project is carried out within the Non-Destructive Assay Working Group of the European Safeguards Research and Development Association (ESARDA). In this study we analyze medium resolution spectra of $U$ and $P u$ standards with the aim to develop an isotopic composition determination algorithm, particularly suited for these types of detectors. We show how the peak shape of a CdZnTe detector is influenced by the instrumentation parameters. The experimental setup consisted of a $500 \mathrm{~mm}^{3} \mathrm{CdZnTe}$ detector, a $2 \times 2$ inch $\mathrm{LaBr}_{3}$ detector, two types of measurement instrumentation - an analogue one and a digital one, and a set of certified samples $-a^{207} \mathrm{Bi}$ point source and $U$ and Pu CBNM standards. The results of our measurements indicate that the lowest contribution to the peak asymmetry and thus the smallest impact on the resolution of the $500 \mathrm{~mm}^{3} \mathrm{CdZnTe}$ detector was achieved with the digital MCA. Analysis of acquired spectra allowed to reject poor quality measurement runs and produce summed spectra files with the least impact of instrumentation instabilities. This work is preliminary to further studies concerning the development of an isotopic composition determination algorithm particularly suited for $\mathrm{CZT}$ and $\mathrm{LaBr}_{3}$ detectors for safeguards applications.
\end{abstract}

Index Terms-CdZnTe, $\mathrm{LaBr}_{3}$, uranium enrichment, isotopic composition, medium resolution, safeguards.

\section{INTRODUCTION}

NTEREST in the use of detectors based on cadmium zinc telluride (CZT, room temperature compound semiconductor) and $\mathrm{LaBr}_{3}$ (room temperature scintillator) as X-ray and gammaray spectrometers has grown rapidly in recent years ${ }^{1,2,3,4}$. CZT detectors are characterized by a wide energy band gap $\left(\mathrm{E}_{\mathrm{g}} \sim 1.6\right.$

I. Meleshenkovskii is with the Nuclear Research Center SCK-CEN, B-2400 Mol, Belgium (e-mail: yaroslav.meleshenkovskii@sckcen.be) and Université libre de Bruxelles, Service de Métrologie Nucléaire (CP/165/84).

A. Borella, K. Van der Meer and M. Bruggeman are with the Nuclear Research Center SCK-CEN, B-2400 Mol, Belgium, (e-mail: alessandro.borella@sckcen.be, michel.bruggeman@sckcen.be).
$\mathrm{eV}$ ) allowing room temperature operation of these detectors, their high atomic number $\left(Z_{\max }=52\right)$ yields a high intrinsic efficiency of gamma absorption (and thus a reduced size of the detector $^{5}$. CZT detectors have now been optimized in their design yielding resolution less than $2 \%$ at $661 \mathrm{keV}\left({ }^{137} \mathrm{Cs}\right)$ for a $10 \mathrm{~mm} \times 10 \mathrm{~mm} \times 5 \mathrm{~mm}$ device ${ }^{6}$. $\mathrm{LaBr}_{3}$ detector has a high light output ( $\sim 60000$ photons/MeV), fast response (decay constant $<30 \mathrm{~ns})$ and shows good energy resolution $(\sim 3 \%$ at $662 \mathrm{keV}$ ${ }^{137} \mathrm{Cs}$ ). Finally, the spectral resolution of these detectors is significantly better in comparison with other room temperature detectors, as shown in figure 1 .

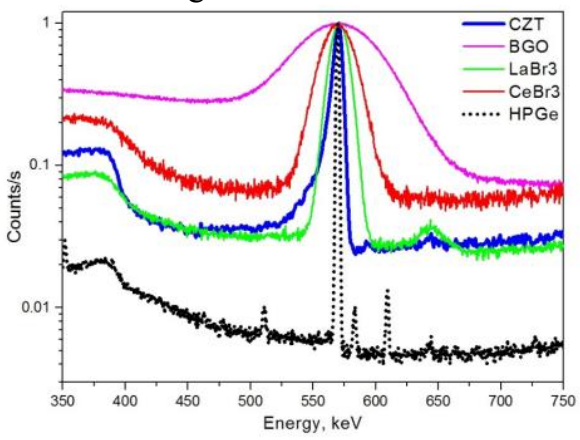

Fig. 1. Comparison of detector resolution for different detectors with $\mathrm{a}^{207} \mathrm{Bi}$ source.

Nuclear safeguards is one of the applications for these detectors because these detectors present a viable alternative to $\mathrm{HpGe}$ detectors used for quantitative gamma ray spectroscopy of uranium samples, plutonium samples and spent fuel assemblies ${ }^{7,8,9}$. Although neither CZT nor $\mathrm{LaBr}_{3}$ detectors provide the resolution as that of $\mathrm{HpGe}$ detectors, they have certain attractive performance features, such as portability, compact design and absence of cryogenics, which make them advantageous for safeguards applications.

Safeguards applications of these detectors require a suitable algorithm for uranium and plutonium isotopic composition determination that would incorporate the spectral particularities of these detectors. Namely, CZT detectors exhibit a peak asymmetry due to incomplete charge collection processes and

N. Pauly and P.E. Labeau, P are with Université libre de Bruxelles, Service de Métrologie Nucléaire (CP/165/84), (e-mail: nipauly@ulb.ac.be, pelabeau@ulb.ac.be)

P. Schillebeeckx is with European Commission Joint Research Center, Retieseweg 111, B-2440 Geel, Belgium, (e-mail: Peter.SCHILLEBEECKX@ec.europa.eu). 
for both detectors a high degree of peak overlapping in complex spectra, such as that of plutonium, is a common issue.

To develop such an algorithm it is necessary to investigate the spectrometric performance of these detectors in a wide energy range and acquire spectra of certified uranium and plutonium standards in order to further test and validate the algorithm. The former requires a detector characterization procedure with point sources where peaks are well separated, which allows to quantitatively describe the peak shapes at different energies with a mathematical model and parametrize it $^{10}$. The latter requires measurements with certified uranium and plutonium standards the enrichment or isotopic composition of which are known. However, both routines can be subject to bias introduced by the instrumentation, which may affect both the quality of the characterization procedure and the acquired spectra of standards.

In this paper we first investigate the influence of instrumentation parameters on the spectrometric performance of a $500 \mathrm{~mm}^{3} \mathrm{CZT}$ detector, and then perform a routine that aims to acquire high quality spectra of uranium and plutonium standards in different measurement configurations. Specifically, the impact of the shaping time constant on the spectral resolution and low-energy tailing was investigated.

This work is in the framework of the development of a uranium and plutonium isotopic composition determination algorithm for CZT and $\mathrm{LaBr}_{3}$ detectors for safeguards applications.

The current manuscript is organized as follows. In section 2 the methodology, detectors, overview of standards and key instrumentation settings that influence the peak shape are given, section 3 provides the results and section 4 concludes the paper.

\section{MATERIALS AND METHODS}

\section{A. Methodology}

As discussed in the literature ${ }^{11}$, there are three major factors that influence the peak shape of a detector: the statistics of the charge creation processes (1); the completeness of the charge collection processes (2) and electronic noise (3). The first of these contributions is inherent to each detector material. The second contribution to the resulting peak shape is a characteristic of each detector and depends primarily on the mobility-lifetime properties of charge carriers (electrons and holes) and resulting charge collection efficiency. Incomplete charge collection efficiency creates a low-energy asymmetry and may cause serious distortion to the peak shape. The third contribution due to the electronic noise depends on the leakage current of the detector and its capacitance.

Although, there exist different designs of CZT detectors that aim to minimize the second contribution, such as co-planar grid, steering grid, pixelated and strip designs ${ }^{12}$, the incomplete charge collection remains a problem for medium to large volume CZT detectors. However, the influence of electronic contribution to the peak asymmetry can be minimized by choosing an appropriate pulse shaping time constant and by operating the detector at the high-voltage bias ${ }^{13}$ recommended by the manufacturer.
The resolution of $\mathrm{LaBr}_{3}$ detectors is approaching that of some CZT detectors and its peaks do not exhibit an asymmetrical shape ${ }^{14}$. Due to this reason, in the current paper we focus on choosing an appropriate pulse shaping time constant for the CZT detector.

To quantitatively characterize the spectroscopic performance of the CZT detector with different pulse shaping time constants we evaluated the following parameters: energy resolution and peak shape parameter values. The full-width at half maximum (FWHM) of the full-energy peaks was assumed as a measure of the energy resolution of the detector. To allow for quantitative analysis of the pulse shaping time constant influence on the low energy tail, we evaluated the full width at $1 / 10$ th of the maximum (FWTM) and the ratio FWTM/FWHM and compared it to that of an ideal Gaussian peak $($ FWTM/FWHM gaussian $=1.82)$. We evaluated the mentioned parameters for the ROI representing the $569.7 \mathrm{keV}$ gamma line of the ${ }^{207} \mathrm{Bi}$ source. The spectra were acquired on two types of measurement instrumentation - a digital one and an analogue one.

As suggested in the literature ${ }^{11}$, to monitor the impact of instrumentation instabilities on the spectra quality we measured uranium and plutonium standards in runs of 7200 seconds each. The total number of measurement runs was selected based upon a criterion to acquire no less than $10^{5}$ counts in corresponding ROI for each of the standards after summing assuming rejected runs. Such methodology allows to produce a high quality summed spectrum file for each sample. To allow in-depth analysis of the quality of uranium and plutonium spectra measured on the CZT and $\mathrm{LaBr}_{3}$ detectors we evaluated the following parameters for the ROIs representing the $185.7 \mathrm{keV}$ gamma line of ${ }^{235} \mathrm{U}$ and $414.3 \mathrm{keV}$ gamma line of ${ }^{239} \mathrm{Pu}$ for each of the measurement runs: peak centroid, FWHM, net peak area and net peak area uncertainty.

As indicated in the reference ${ }^{15}$ : "The peak centroid is calculated as the sum of the background corrected channel contents multiplied by the channel number divided by the sum of the background corrected channel contents in the range of the channels above the photopeak half maximum":

$$
\text { Centroid }=\frac{\sum_{i=l}^{h} i * S p_{i}}{\sum_{i=l}^{h} S p_{i}}
$$

Where $i$ is the channel number, $l$ is the lowest channel above the half maximum, $h$ is the highest channel above the half maximum and $S p_{i}$ are the net contents of channel $i$.

As indicated in the reference ${ }^{15}$ : "The net peak area (1) is calculated as the number of counts above the background in a specified ROI. The background area (2) is determined by averaging 4 points on both sides of the peak (the ROI limit points and 3 outer points) and linear fit":

$$
\begin{aligned}
& N P A=I n t-B K G \\
& B K G=\frac{R O I_{e}-R O I_{b}+1}{8}\left(\sum_{i=R O I_{b}-3}^{R O I_{b}} S p_{i}+\sum_{i=R O I_{e}}^{R O I_{e}+3} S p_{i}\right)
\end{aligned}
$$




$$
\text { Int }=\sum_{i=R O I_{b}}^{R O I_{e}} S p_{i}
$$

Where $N P A$ is the net peak area, $B K G$ is the background, Int is the ROI's integral, $S p_{i}$ is the absolute number of counts in channels $i, R O I_{b}$ is the ROI's start channel, $R O I_{e}$ is the ROI's stop channel and $i$ is the channel number.

As indicated in the reference ${ }^{15}$ : "The Full Width at Half Maximum (FWHM) is calculated as the background corrected photopeak's width at one-half of its maximum amplitude. The algorithm first proceeds down one side of the peak until it finds a channel in which counts fall below the half maximum channel. The same algorithm is applied for the other side of the peak. The left and the right FWHM points are the interpolated channels between the counts of the channel below and above the half maximum value". The same algorithm was used to determine the FWTM.

\section{B. Detectors}

The CZT detector used was a $10 \mathrm{~mm} \times 10 \mathrm{~mm} \times 5 \mathrm{~mm}$ device of a hemispheric design fabricated by RITEC (Latvia) as shown in figure 2. The high-voltage bias applied to the detector was $1400 \mathrm{~V}$.

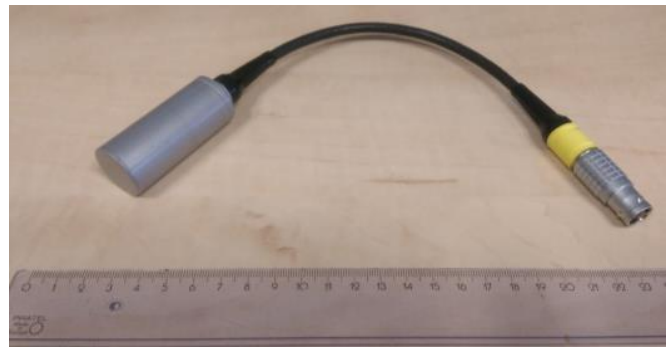

Figure 2: $500 \mathrm{~mm}^{3} \mathrm{CZT}$ detector.

The $\mathrm{LaBr}_{3}$ scintillator used was a $2 \times 2$ inch device fabricated by Saint-Gobain Crystals (France) as shown in figure 3 . The high-voltage bias applied to the detector was $590 \mathrm{~V}$.

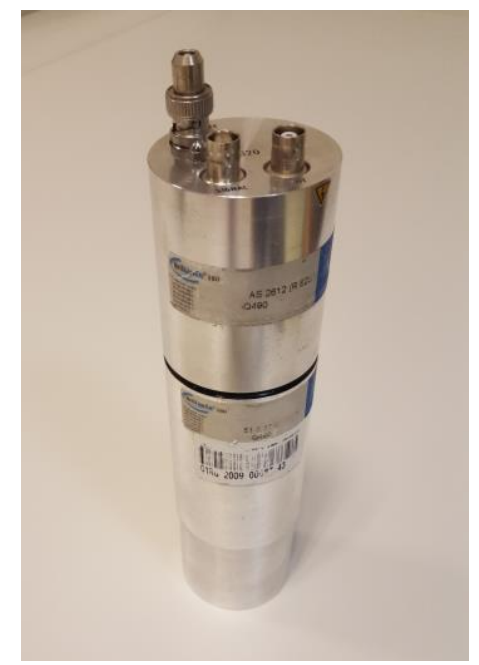

Figure 3: $2 \times 2$ inch $\mathrm{LaBr}_{3}$ scintillator.

\section{Electronics}

The analogue electronics used to measure spectra consisted of standard nuclear spectroscopy signal processing chain (charge sensitive preamplifier, linear amplifier and shaper, analogue-to-digital converter) and a data acquisition software DAQ2000 developed by LabView. The schematic diagram of the analogue instrumentation circuitry is shown in figure 4.

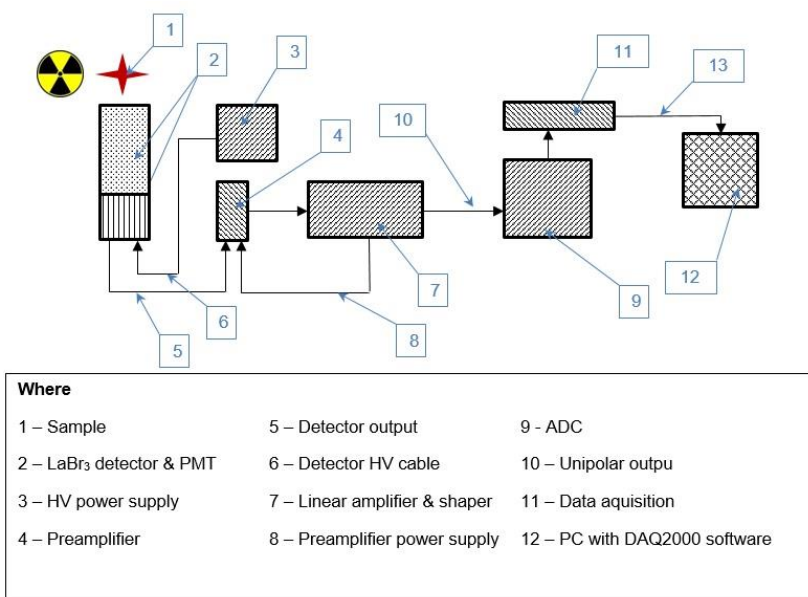

Figure 4: Schematics of the analogue instrumentation circuitry.

The digital instrumentation consisted of a digital multichannel-analyzer (MCA) module, model 527, produced by GBS-Elektronik $^{15}$ (Germany) and a data acquisition software WinSpec $^{16}$. The schematic diagram of the digital instrumentation circuitry is shown in figure 5.

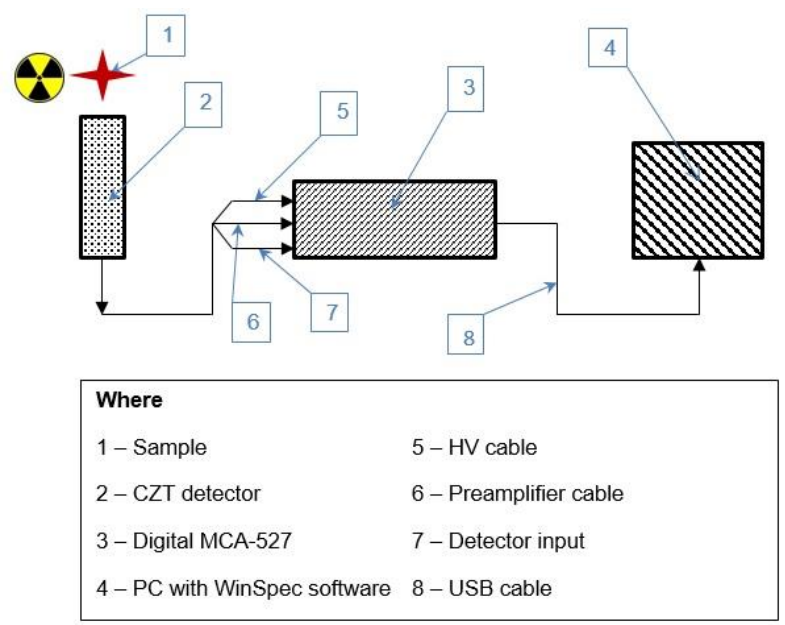

Figure 5: Schematics of the digital instrumentation circuitry.

The analogue instrumentation allowed to vary the pulse shaping time constant in the following range of values: 0.25 ; $0.5 ; 1 ; 2 ; 4$ and $8 \mu \mathrm{s}$. The digital instrumentation allowed to vary the pulse shaping time constant in a wide range as well, however in the current paper we investigated the spectrometric performance of the CZT detector at the following values: 0.7 and $1.2 \mu \mathrm{s}$.

\section{Samples}

To characterize the spectroscopic performance of the CZT detector we used a certified ${ }^{207} \mathrm{Bi}$ source $(569.7 ; 1063$ and 1770 $\mathrm{keV}$ gamma lines), as shown in figure 6 . 


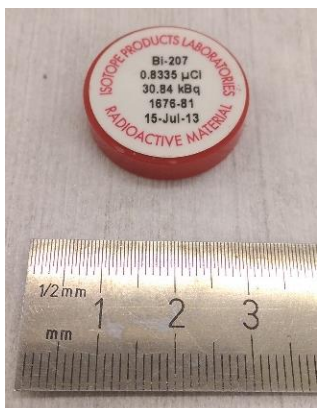

Figure 6: ${ }^{207} \mathrm{Bi}$ certified source.

For uranium measurements we used certified uranium CBNM (Central Bureau for Nuclear Measurements) standards, as shown in figure 7 , of five different enrichment values: $0.31 \%, 0.71 \%, 1.94 \%, 2.95 \%$ and $4.46 \%$.

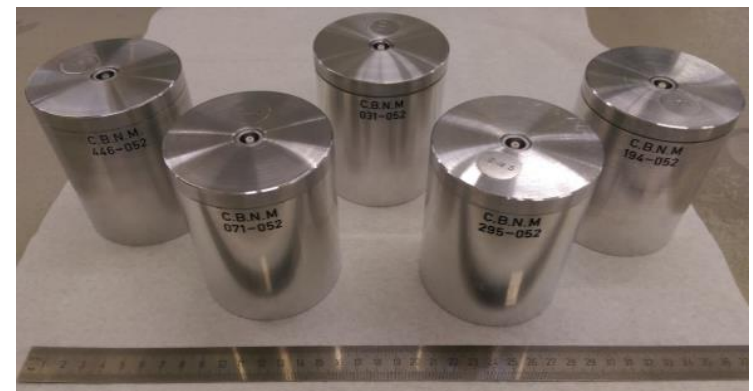

Figure 7: Uranium certified standards.

For plutonium measurements we used certified plutonium CBNM standards, as shown in figure 8, of four different isotopic compositions as indicated in table 1 .

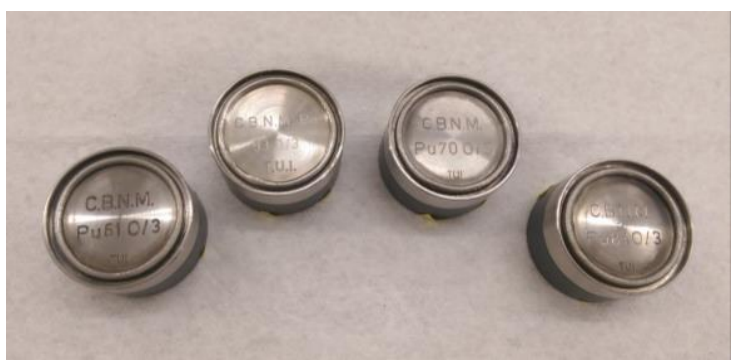

Figure 8: Plutonium certified standards.

\begin{tabular}{|c|c|c|c|c|c|c|}
\hline \multirow{2}{*}{$\begin{array}{c}\text { CBNM } \\
\text { Sample }\end{array}$} & \multicolumn{7}{|c|}{ Mass percent* } \\
\cline { 2 - 7 } & ${ }^{238} \mathrm{Pu}$ & ${ }^{239} \mathrm{Pu}$ & ${ }^{240} \mathrm{Pu}$ & ${ }^{241} \mathrm{Pu}$ & ${ }^{242} \mathrm{Pu}$ & ${ }^{241} \mathrm{Am} / \mathrm{Pu}$ \\
\hline $\mathrm{Pu} 93$ & 0.0117 & 93.4123 & 6.3131 & 0.2235 & 0.0395 & 0.1047 \\
\hline $\mathrm{Pu} 84$ & 0.0703 & 84.3377 & 14.2069 & 1.0275 & 0.3576 & 0.2173 \\
\hline $\mathrm{Pu} 70$ & 0.8458 & 73.3191 & 18.2945 & 5.4634 & 2.0772 & 1.1705 \\
\hline $\mathrm{Pu} 61$ & 1.1969 & 62.5255 & 25.4058 & 6.6793 & 4.1925 & 1.4452 \\
\hline
\end{tabular}

Table 1: Isotopic composition of plutonium standards (*on 1986).

\section{E. Collimators \& Attenuators}

In order to reduce the impact of scattered photons reaching the detector from the sample and nearby materials, the measurements were conducted with the lead collimators, the exact dimensions of which for CZT and $\mathrm{LaBr}_{3}$ detectors are shown in figures $9 \& 10$ respectively. The uranium samples were positioned directly on the top surface of the lead collimators for which there were bored seats $81 \mathrm{~mm}$ in diameter and $5 \mathrm{~mm}$ deep. The sample-to-detector distance was $20 \mathrm{~mm}$.

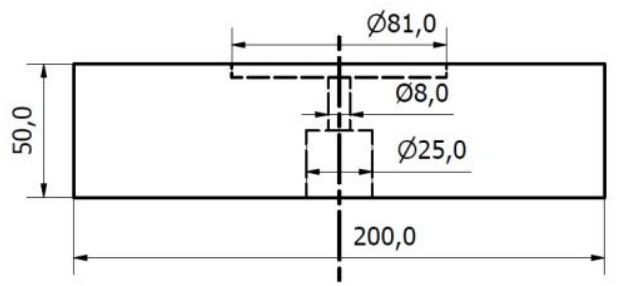

Figure 9: CZT lead collimator.

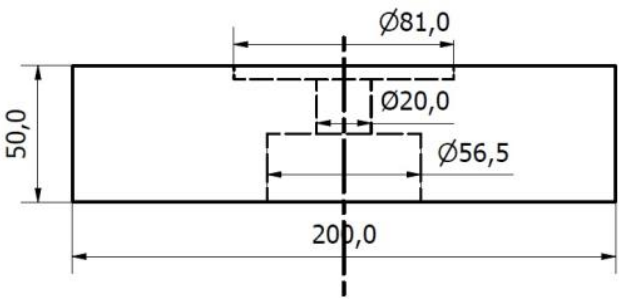

Figure 10: $\mathrm{LaBr}_{3}$ lead collimator.

Measurements of plutonium certified standards were conducted in two configurations - with and without lead collimators on both detectors to investigate their impact on the spectra quality. Plutonium standards were positioned in adjustable height holders above the detectors. To reduce the impact of ${ }^{241} \mathrm{Am} 59 \mathrm{keV}$ gamma line on plutonium spectra we used a set of cadmium (1.5 mm thick) and copper $(0.5 \mathrm{~mm}$ thick) attenuators, as shown in figure 11:

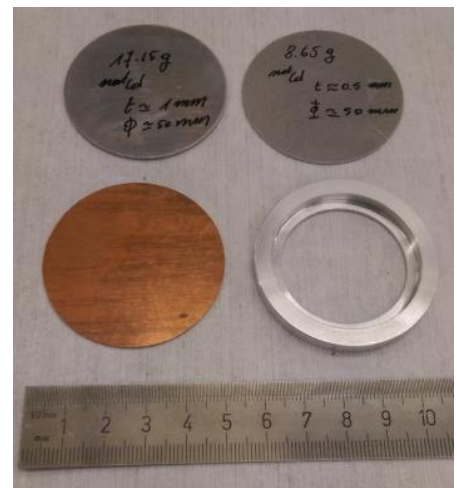

Figure 11: Cadmium and copper attenuators.

\section{RESULTS AND DISCUSSION}

\section{A. Spectroscopic Performance of CZT Detector}

We first performed spectroscopic performance assessment of the CZT detector at different pulse shaping time constants on both types of instrumentation for the background corrected ROI corresponding to the $569.7 \mathrm{keV}$ gamma line of the ${ }^{207} \mathrm{Bi}$ source. We measured the ${ }^{207} \mathrm{Bi}$ source for 3600 seconds (real time) on both types of instrumentation. The spectrometric performance of the CZT detector at various pulse shaping time constants for the analogue and digital instrumentation is shown in figures 12 and 13 respectively. 


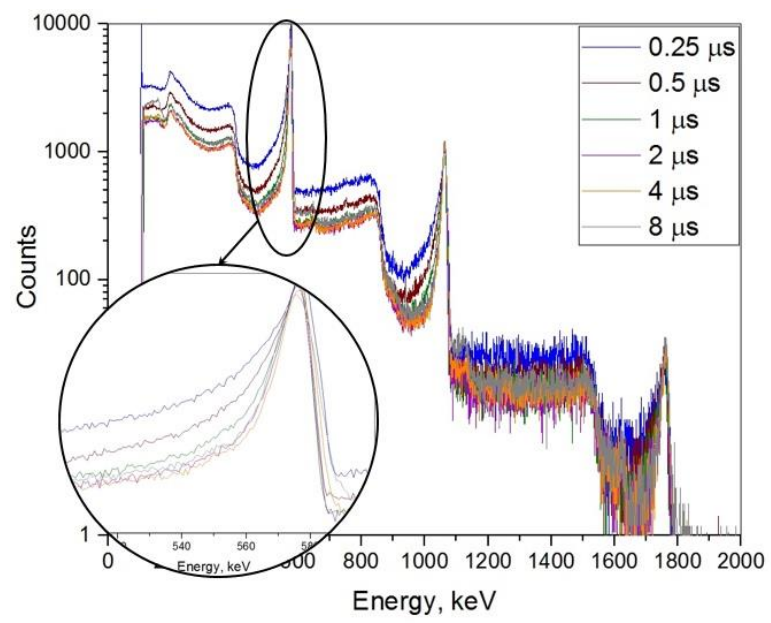

Figure 12: Spectrometric performance of the $500 \mathrm{~mm}^{3} \mathrm{CZT}$ detector, analogue instrumentation.

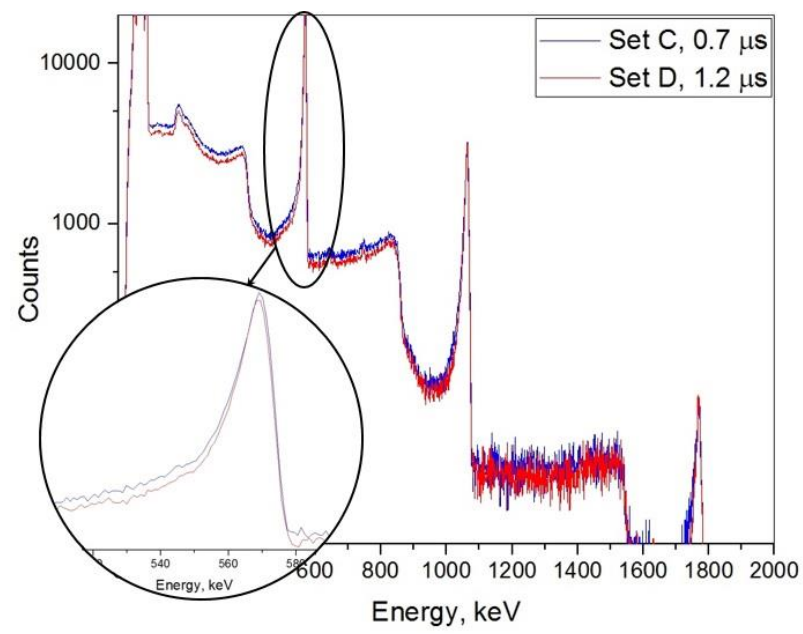

Figure 13: Spectrometric performance of the $500 \mathrm{~mm}^{3} \mathrm{CZT}$ detector, digital MCA.

Results indicate that performance improvement for the CZT detector on analogue instrumentation is achieved starting from $1 \mu \mathrm{s}$ pulse shaping time constant value, however there is no significant improvement in performance for values exceeding 2 $\mu$ s. Indeed, at $2 \mu$ s pulse shaping time constant on analogue instrumentation the impact on the tailing is reduced by $56 \%$ given by the peak shape parameter, compared to the results for $0.25 \mu$ s pulse shaping time constant, as indicated in table 2 .

\begin{tabular}{|c|c|c|c|}
\hline $\begin{array}{c}\text { Pulse shaping } \\
\text { constant, } \\
\mu \text { s }\end{array}$ & FWHM, \% & FWTM, \% & $\begin{array}{c}\text { Peak shape } \\
\text { parameter } \\
\text { (FWTM/ } \\
\text { FWHM) }\end{array}$ \\
\hline 0.25 & 2.01 & 8.04 & 4 \\
\hline 0.5 & 1.54 & 5.10 & 3.3 \\
\hline 1 & 1.59 & 4.46 & 2.8 \\
\hline 2 & 1.38 & 3.11 & 2.25 \\
\hline 4 & 1.28 & 2.89 & 2.25 \\
\hline 8 & 1.26 & 2.69 & 2.13 \\
\hline
\end{tabular}

Table 2: Spectrometric performance parameters for the $569.7 \mathrm{keV}$ ROI of the ${ }^{207} \mathrm{Bi}$ source, analogue instrumentation.

Results of the spectrometric performance for the CZT detector on digital MCA indicate that optimal performance is achieved with $1.2 \mu$ s pulse shaping time constant value, as indicated in table 3. During the spectrometric performance assessment of the CZT detector with the digital MCA the settings of digital filters were kept constant.

\begin{tabular}{|c|c|c|c|}
\hline $\begin{array}{c}\text { Pulse shaping } \\
\text { constant, } \\
\mu \mathrm{s}\end{array}$ & FWHM, \% & FWTM, \% & $\begin{array}{c}\text { Peak shape } \\
\text { parameter } \\
\text { (FWTM/ } \\
\text { FWHM) }\end{array}$ \\
\hline 0.7 & 1.45 & 3.31 & 2.28 \\
\hline 1.2 & 1.27 & 2.71 & 2.12 \\
\hline
\end{tabular}

Table 3: Spectrometric performance parameters for the $569.7 \mathrm{keV}$ ROI of the ${ }^{207} \mathrm{Bi}$ source, digital MCA.

Comparison of digital and analogue instrumentation performance between each other for the $569.7 \mathrm{keV}$ ROI of the

${ }^{207} \mathrm{Bi}$ source is given in figure 14 . Indeed, it is evident that the digital MCA with 1.2 $\mu$ s pulse shaping time constant exhibits less electronic impact on the resolution and tailing of the CZT detector peak shape.

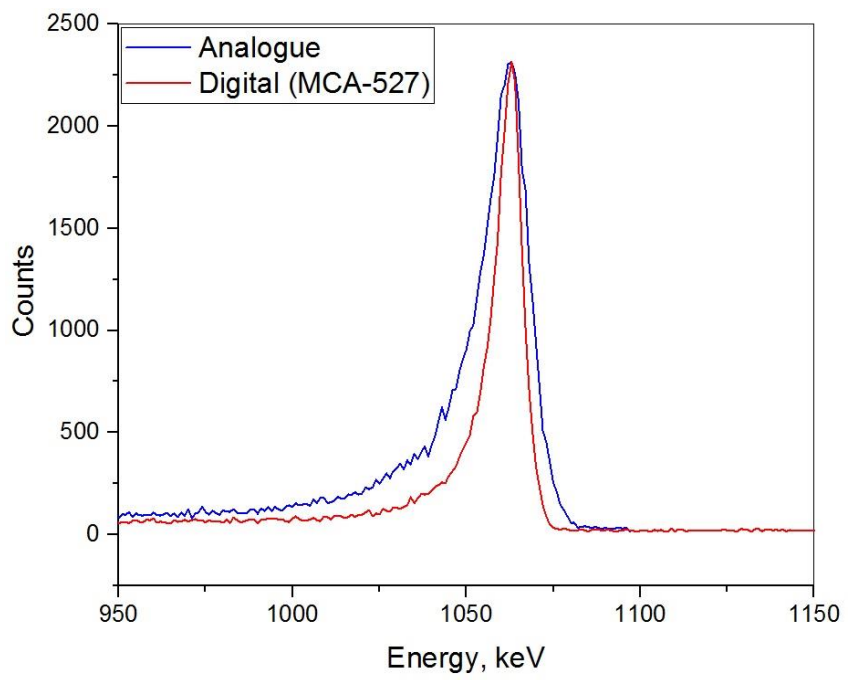

Figure 14: Digital and analogue spectroscopic performance comparison, ROI $569.7 \mathrm{keV}$ of the ${ }^{207} \mathrm{Bi}$ source.

\section{B. Stability of CZT and $\mathrm{LaBr}_{3}$ Detectors}

For the measurements of uranium and plutonium standards we used $1.2 \mu$ s pulse shaping time constant for the CZT detector on the digital MCA and $0.25 \mu$ s pulse shaping time constant for the $\mathrm{LaBr}_{3}$. These settings allowed to achieve good throughput of the analogue pulse processing chain coupled with the $\mathrm{LaBr}_{3}$ detector considering its high efficiency and to minimize the deadtime to less than $2 \%$ on the CZT detector coupled with the digital MCA. The sample-to-detector distances used for plutonium measurements are given in table 4 .

To investigate the time stability of the CZT and $\mathrm{LaBr}_{3}$ detectors during the measurements of uranium and plutonium standards we evaluated the following parameters for each measurement run: peak centroid, FWHM, net peak area and net peak area uncertainty, as shown, for example, for the CBNM-031 uranium sample in figures 15, 16 and 17 respectively. For this purpose, the corresponding Matlabbased programs were developed from scratch; they accept the 
raw spectra files and the user defined ROI. Identical ROI limits were used for all measurement runs.

\begin{tabular}{|c|c|c|c|c|}
\hline \multirow{2}{*}{ CBNM Sample } & \multicolumn{3}{|c|}{ Sample-to-detector distance, cm } \\
\cline { 2 - 5 } & \multicolumn{2}{|c|}{ LaBr $_{3}$} & \multicolumn{2}{c|}{ CZT } \\
\cline { 2 - 5 } & Lead & No lead & Lead & No lead \\
\hline Pu93 & 25 & 25 & 5.4 & 4 \\
\hline Pu84 & 25 & 25 & 5.4 & 4 \\
\hline Pu70 & 25 & 25 & 8 & 8 \\
\hline Pu61 & 25 & 25 & 8 & 8 \\
\hline
\end{tabular}

Table 4: Sample-to-detector distance for plutonium measurements.

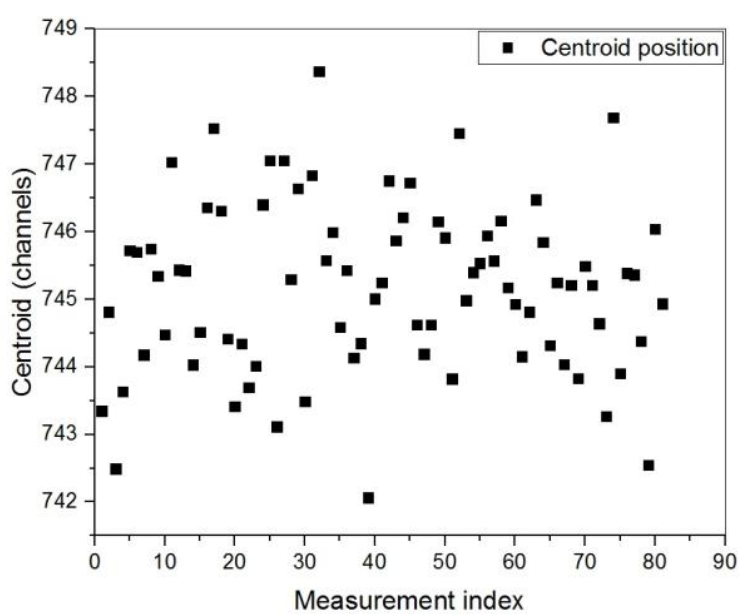

Figure 15: CBNM-031 sample centroid positions.

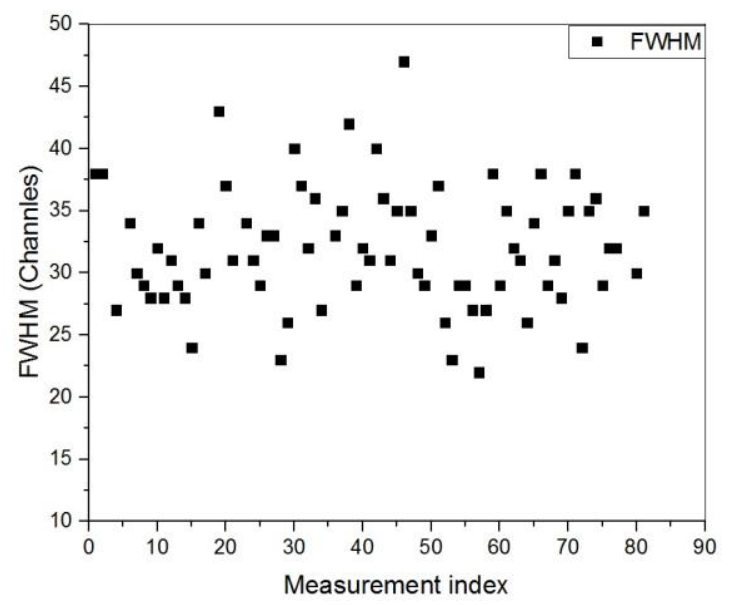

Figure 16: CBNM-031 sample peak widths.

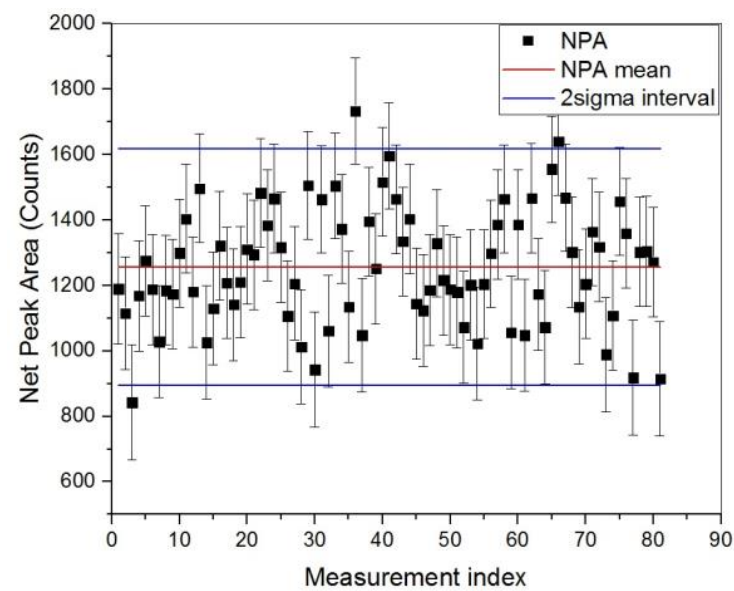

Figure 17: CBNM-031 sample net peak areas and uncertainties.

In order to reject poor quality spectra the rejection criteria based on the $95.5 \%$ confidence interval for the net peak area values, \pm 1 channel centroid drift for the $185.7 \mathrm{keV}$ gamma-line of ${ }^{235} \mathrm{U}$ and \pm 2 channels centroid position drift for the $413.4 \mathrm{keV}$ gamma-line of ${ }^{239} \mathrm{Pu}$ were adopted. For each standard measured we calculated the mean value of the observed net peak area values for all measurement runs and set the minimum and maximum limits for the net peak area values in corresponding ROIs based on the two sigma interval around that mean value. Those files whose observed net peak area values fall out of this interval, as well as spectra files with significant centroid position drift in corresponding ROIs, were rejected. Spectra that satisfy the criteria were used to produce the summed spectrum file for each given standard. The statistical details of the summed spectra files for all CBNM standards measured are given in tables 5, 6, 7, 8, 9 and 10 respectively.

\begin{tabular}{|c|c|c|c|c|c|}
\hline & \multirow{2}{*}{$\begin{array}{c}\text { Total } \\
\text { CBNM }\end{array}$} & N. r. & \multirow{2}{*}{$\begin{array}{c}\text { Cor. } \mathrm{m} . \\
\text { time, } \\
\text { Sample }\end{array}$} & $\mathrm{suns}$ & \multicolumn{2}{|c|}{$\begin{array}{c}\text { spectra (ROI 185.7 } \\
\text { keV) }\end{array}$} \\
\cline { 5 - 6 } & & & & $\begin{array}{c}\text { NPA, } \\
\text { counts }\end{array}$ & $\begin{array}{c}\text { NPA } \\
\text { unc., } \delta\end{array}$ \\
\hline U031 & 583200 & 36 & 324000 & 56585 & 1.9 \\
\hline U071 & 410400 & 9 & 345600 & 129620 & 0.8 \\
\hline U194 & 345600 & 4 & 316800 & 319203 & 0.4 \\
\hline U295 & 223200 & 2 & 208800 & 315350 & 0.3 \\
\hline U446 & 151200 & 1 & 144000 & 332037 & 0.3 \\
\hline
\end{tabular}

Table 5: CZT summed uranium spectra results. 


\begin{tabular}{|c|c|c|c|c|c|}
\hline & \multirow{2}{*}{$\begin{array}{c}\text { Total } \\
\text { CBNM } \\
\text { Sample }\end{array}$} & $\begin{array}{c}\text { N. r. } \\
\mathrm{s}\end{array}$ & \multirow{2}{*}{$\begin{array}{c}\text { Cor. } \mathrm{m} . \\
\text { runs }\end{array}$} & \multicolumn{2}{|c|}{$\begin{array}{c}\Sigma \text { spectra (ROI } 185.7 \\
\text { keV) }\end{array}$} \\
\cline { 5 - 6 } & 338400 & 33 & 100800 & $\begin{array}{c}\text { NPA, } \\
\text { counts }\end{array}$ & $\begin{array}{c}\text { NPA } \\
\text { unc., } \delta\end{array}$ \\
\hline U031 & 3399278 & 0.5 \\
\hline U071 & 165600 & 4 & 136800 & 3510569 & 0.2 \\
\hline U194 & 64800 & 2 & 50400 & 3523567 & 0.1 \\
\hline U295 & 28800 & 0 & 28800 & 2291632 & 0.1 \\
\hline U446 & 21600 & 0 & 21600 & 2311297 & 0.1 \\
\hline
\end{tabular}

Table 6: $\mathrm{LaBr}_{3}$ summed uranium spectra results.

\begin{tabular}{|c|c|c|c|c|c|}
\hline \multirow{2}{*}{$\begin{array}{l}\text { CBNM } \\
\text { Sample }\end{array}$} & \multirow{2}{*}{$\begin{array}{l}\text { Total } \\
\text { m. time, } \\
\text { s }\end{array}$} & \multirow{2}{*}{$\begin{array}{l}\text { N. r. } \\
\text { runs }\end{array}$} & \multirow{2}{*}{$\begin{array}{l}\text { Cor. } \mathrm{m} . \\
\text { time,s }\end{array}$} & \multicolumn{2}{|c|}{$\begin{array}{c}\Sigma \text { spectra (ROI } 413.4 \\
\mathrm{keV})\end{array}$} \\
\hline & & & & $\begin{array}{l}\text { NPA, } \\
\text { counts }\end{array}$ & $\begin{array}{c}\text { NPA } \\
\text { unc. } \delta\end{array}$ \\
\hline Pu93 & 280800 & 2 & 266400 & 9979283 & 0.06 \\
\hline Pu84 & 172800 & 2 & 158400 & 5299094 & 0.09 \\
\hline Pu70 & 352800 & 2 & 338400 & 1901123 & 0.2 \\
\hline Pu61 & 331200 & 3 & 309600 & 1436089 & 0.3 \\
\hline
\end{tabular}

Table 7: CZT summed plutonium spectra results, without lead.

\begin{tabular}{|c|c|c|c|c|c|}
\hline & \multirow{2}{*}{$\begin{array}{c}\text { Total } \\
\text { CBNM }\end{array}$} & \multirow{2}{*}{$\begin{array}{c}\text { N. r. } \\
\text { m. time, } \\
\text { runs }\end{array}$} & \multirow{2}{*}{$\begin{array}{c}\text { Cor. } \mathrm{m} . \\
\text { time, }\end{array}$} & \multicolumn{2}{|c|}{$\begin{array}{c}\text { k spectra (ROI 413.4 } \\
\text { keV) }\end{array}$} \\
\cline { 5 - 6 } & & & & $\begin{array}{c}\text { NPA, } \\
\text { counts }\end{array}$ & $\begin{array}{c}\text { NPA } \\
\text { unc., } \delta\end{array}$ \\
\hline Pu93 & 172800 & 1 & 165600 & 2174818 & 0.1 \\
\hline Pu84 & 172800 & 0 & 172800 & 1982828 & 0.1 \\
\hline Pu70 & 172800 & 1 & 165600 & 587762 & 0.3 \\
\hline Pu61 & 172800 & 1 & 165600 & 511253 & 0.4 \\
\hline
\end{tabular}

Table 8: CZT summed plutonium spectra results, with lead.

\begin{tabular}{|c|c|c|c|c|c|}
\hline & \multirow{2}{*}{$\begin{array}{c}\text { Total } \\
\text { CBNM } \\
\text { Sample }\end{array}$} & \multirow{2}{*}{$\begin{array}{c}\text { N. r. } \\
\mathrm{s}\end{array}$} & \multirow{2}{*}{$\begin{array}{c}\text { Cor. } \mathrm{m} . \\
\text { runs }\end{array}$} & \multicolumn{2}{|c|}{$\begin{array}{c}\Sigma \text { spectra (ROI 413.4 } \\
\text { keV) }\end{array}$} \\
\cline { 5 - 6 } & & & $\begin{array}{c}\text { NPA, } \\
\text { counts }\end{array}$ & $\begin{array}{c}\text { NPA } \\
\text { unc., } \delta\end{array}$ \\
\hline Pu93 & 57600 & 3 & 36000 & 3126014 & 0.1 \\
\hline Pu84 & 79200 & 2 & 64800 & 4964420 & 0.1 \\
\hline Pu70 & 57600 & 2 & 43200 & 3447786 & 0.2 \\
\hline Pu61 & 28800 & 0 & 28800 & 1704848 & 0.3 \\
\hline
\end{tabular}

Table 9: $\mathrm{LaBr}_{3}$ summed plutonium spectra results, without lead.

\begin{tabular}{|c|c|c|c|c|c|}
\hline & \multirow{2}{*}{$\begin{array}{c}\text { Total } \\
\text { Sample }\end{array}$} & \multirow{2}{*}{$\begin{array}{c}\text { N. t. } \\
\mathrm{s}\end{array}$} & \multirow{2}{*}{$\begin{array}{c}\text { Cor. } \mathrm{m} . \\
\text { time, }\end{array}$} & \multicolumn{2}{|c|}{$\begin{array}{c}\text { k spectra (ROI 413.4 } \\
\text { keV) }\end{array}$} \\
\cline { 5 - 6 } & & & & $\begin{array}{c}\text { NPA, } \\
\text { counts }\end{array}$ & $\begin{array}{c}\text { NPA } \\
\text { unc., } \delta\end{array}$ \\
\hline Pu93 & 72000 & 4 & 43200 & 1079943 & 0.2 \\
\hline Pu84 & 21600 & 0 & 21600 & 494498 & 0.4 \\
\hline Pu70 & 28800 & 3 & 7200 & 145344 & 0.9 \\
\hline Pu61 & 21600 & 0 & 21600 & 407703 & 0.5 \\
\hline
\end{tabular}

Table 10: $\mathrm{LaBr}_{3}$ summed plutonium spectra results, with lead.

Where Total m. time is the total measurement time, N. r. runs is the number of rejected runs, Cor. M. time is the corrected measurement time and NPA is the net peak area.

\section{CONCLUSION}

Measurements of uranium and plutonium certified standards were conducted on two detectors - a $500 \mathrm{~mm}^{3}$ CZT semiconductor detector and a $2 \times 2$ inch $\mathrm{LaBr}_{3}$ scintillator.

Asymmetrical peak shape of a CZT detector can be quite sensitive to the electronic noise introduced by the pulse processing chain. This can negatively influence the quality of acquired spectra. To reduce the impact of the electronic noise on the spectra quality we first investigated the spectrometric performance of a $500 \mathrm{~mm}^{3} \mathrm{CZT}$ detector of hemispheric design at different settings of the pulse shaping time constant on two types of instrumentation - a digital one and an analogue one. The results indicated that asymmetrical peak shape of a CZT detector is very sensitive to the pulse shaping time constant value and best results were achieved with the digital MCA. The $\mathrm{LaBr}_{3}$ detector does not have an asymmetrical peak shape, however its resolution is worse than that of CZT in the whole range of energies.

To analyze the stability of both detectors during the measurements of uranium and plutonium standards we performed measurements in runs of 7200 seconds each. Such methodology allowed us to reject poor quality spectra and to produce the summed spectra files based on the measurement runs that satisfy the rejection criteria. The results of uranium measurements indicate that both detectors showed quite strong gain drift, especially during the long-term measurements of low enriched uranium standards. The observed fluctuations of the net peak area values in the ROI corresponding to the $185.7 \mathrm{keV}$ gamma-line of ${ }^{235} \mathrm{U}$ were smaller.

The results of plutonium measurements indicate strong gain drift on the $\mathrm{LaBr}_{3}$ detector coupled with the analogue instrumentation. Thus, the number of rejected measurement runs was higher for the $\mathrm{LaBr}_{3}$ detector in comparison with the CZT detector. CZT detector coupled with the digital MCA showed better stability in measurements with and without lead collimators during the measurements of plutonium standards.

The results of this study will be further used for the development of a uranium and plutonium isotopic composition determination algorithm particularly suited for CZT and $\mathrm{LaBr}_{3}$ detectors for safeguards applications. The produced spectra of 
uranium and plutonium standards will be added to the ESARDA database.

\section{REFERENCES}

[1] T.E. Schlesinger, R.B. James (Eds.), Semiconductors for Room Temperature Nuclear Detector Applications, Semiconductors and Semimetals, vol. 43, Academic Press, New York, 1995.

[2] R.B. James, T.E. Schlesinger, P. Siffert, M. Cuzin, M. Squillante, W. Dusi (Eds.), Semiconductors for Room Temperature Nuclear Detector Applications II, in: Materials Research Society Symposium Proceedings, vol. 487, Pittsburgh, MA, 1998.

[3] R.B. James, P. Siffert, Proceedings of the 11th International Workshop on Room Temperature Semiconductor X- and GammaRay detectors and Associated Electronics, Nucl. Instr. and Meth. A 458 (2001) 1.

[4] Raquel Gonzales, "Performance comparison of a large CZT semiconductor detector and $\mathrm{a} \mathrm{LaBr}_{3}(\mathrm{Ce})$ scintillator detector", 2005.

[5] T. Takahashi and S. Watanabe, "Recent Progress in CdTe and CdZnTe Detectors", IEEE Transactions on Nuclear Science, Page(s): 950 - 959 (Volume: 48, Issue: 4, Aug 2001.

[6] Etim Iniobong Prosper et al, "Characterisation of CeriumDoped Lanthanum Bromide scintillation detector", Lat. Am. J. Phys. Educ. Vol. 6, No. 1, March 2012.

[7] V. Ivanov, P. Dorogov "Further development of hemispheric CZT detectors for safaguards applications", Proceedings of the 21 st ESARDA Annual Symposium, Sevilla, Spain, 4-6 May 1999.

[8] Wayne D. Ruhter "Application of CZT detectors in nuclear materials safeguards", Proc. SPIE 3446, Hard X-Ray and Gamma-Ray Detector Physics and Applications, 204 (July 1, 1998); doi:10.1117/12.312892.

[9] V. Ivanov et al, "Performance Evaluation of New Generation CdZnTe Detectors for Safeguards Applications", 2014.

[10] I. Meleshenkovskii et al, "Characterization of a $500 \mathrm{~mm}^{3}$ CdZnTe Detector for $\mathrm{U}$ and $\mathrm{Pu}$ isotopic Composition Determination tasks in Safeguards", ESARDA proceedings, Dusseldorf, Germany, 2017.

[11] K. Debertin, R. G. Helmer, "Emission-rate determinations" in Gamma- and X-ray Spectrometry with Semiconductor Detectors, North-Holland, 1988, ch. 4, pp. 286287 book.

[12] L. Abbene, S. de Sordo, "Spectroscopic response of a CZT multiple electrode detector".

[13] Gordon R. Gilmore, "Electronics for Gamma-Ray Spectrometry" in Practical Gamma-ray Spectrometry, 2nd Edition, Nuclear Training Services Ltd Warrington, UK, John Wiley \& Sons, Ltd, 2008, ch. 4, pp. 73-74.
[14] Kromek Coplanar Grid CZT detectors, brochure, http://www.kromek.com/index.php/products/nuclear-

technology/czt/cpg

[15] MCA-527 user manual, update 2012-08-07, GBSElektronik GmbH, Großerkmannsdorf, Germany, 2012.

[16] https://www.gbs-elektronik.de/en/downloads/downloadsnuclear-measurements.php 